\title{
A NOTE ON GENERATING SETS FOR INVERTIBLE IDEALS ${ }^{1}$
}

\section{ROBERT GILMER}

It is well known that an invertible fractional ideal of a commutative ring with identity must be finitely generated. S. U. Chase has shown that for any positive integer $n$, there is an integral domain $W_{n}$ containing an invertible ideal with a basis of $n$, but no fewer, generators. Chase's example does not appear in the literature, but the example has been referred to by Bass in $[1,541]$, by Swan in $[4,270]$, and by Gilmer and Heinzer in [2]. We know of no verification of the details of Chase's example independent of Swan's results in [4]; these results of Swan are quite involved.

In this note, we give an example of a domain $D_{n}$ with identity containing an invertible ideal $A_{n}$ with a basis of $n$, but no fewer, generators. The domain $D_{n}$ is related to, but not isomorphic to, Chase's domain $W_{n}$. However, our verification that $A_{n}$ has no basis of fewer than $n$ elements depends only upon Lemma 1 , a result which should be of independent interest in itself. The proof of Lemma 1 requires essentially only the Borsuk-Ulam Theorem $[3,152]$.

We use $E$ to denote the field of real numbers; $n$ is a positive integer greater than one, and $\left\{X_{i}\right\}_{i=1}^{n}$ is a set of indeterminates over $E$.

Lemma 1. If $f_{1}, \cdots, f_{n-1} \in E\left[X_{1}, \cdots, X_{n}\right]$, where each nonzero monomial of each $f_{i}$ has odd degree, and if

$$
S^{n}=\left\{u=\left(u_{1}, \cdots, u_{n}\right) \in E^{n} \mid \sum_{i=1}^{n} u_{i}^{2}=\alpha\right\},
$$

where $\alpha$ is a fixed positive real number, then $f_{1}, \cdots, f_{n-1}$ have a common zero on $S^{n}$.

Proof. Consider the mapping $g: S^{n} \rightarrow E^{n-1}$ defined by $g(u)=\left(f_{1}(u)\right.$, $\left.\cdots, f_{n-1}(u)\right) . g$ is continuous, and the hypothesis on the $f_{i}$ 's implies that $g(u)=-g(-u)$ for any $u \in S^{n}$. By the Borsuk-Ulam Theorem, there exists a pair $\{u,-u\}$ of antipodal points of $S^{n}$ such that $g(u)=g(-u)$. Consequently, $g(u)=(0, \cdots, 0)$, and $u$ is a common zero of the $f_{i}^{\prime}$ 's on $S^{n}$.

Now let $J_{n}=E\left[\left\{X_{i} X_{j}\right\}_{1 \leq i, j \leq n}\right]$, let $s=\sum_{i=1}^{n} X_{i}^{2}$, and let $D_{n}=\left(J_{n}\right)_{N}$, where $N=\left\{s^{i}\right\}_{i=0}^{\infty}$. If $A_{n}$ is the ideal of $D_{n}$ generated by $\left\{X_{1} X_{i}\right\}_{1 \leq i \leq n}$,

Received by the editors October 29, 1968.

${ }^{1}$ During the writing of this paper, the author received support from National Science Foundation Grant GP-8424. Communications with Christian U. Jensen and Perrin Wright were helpful in the proof of Lemma 1. 
then $A_{n}$ is invertible, for clearly $A_{n}^{2} \subseteq X_{1}^{2} D_{n}$, but equality holds since $X_{1}^{2}=X_{1}^{2} s / s=\sum_{i=1}^{n}\left(X_{1} X_{i}\right)^{2} / s \in A_{n}^{2}$. Therefore, $A_{n}$ is invertible and has a basis of $n$ elements. But if $g_{1}, \cdots, g_{n-1} \in A_{n}$, then there exist polynomials $f_{1}, \cdots, f_{n-1} \in E\left[X_{1}, \cdots, X_{n}\right]$ such that each nonzero monomial in each $f_{i}$ has odd degree and such that $g_{i}=X_{1} f_{i}$ for each $i$. By Lemma 1 the $f_{i}$ 's, and hence the $g_{i}{ }^{\prime}$, have a common zero $u=\left(u_{1}, \cdots, u_{n}\right)$ in $E^{n}$ other than the origin. The equality $A_{n}$ $=\left\{g_{1}, \cdots, g_{n-1}\right\} D_{n}$ would imply the existence of a subset $\left\{h_{i j}\right\}_{1 \leq i, j \leq n-1}$ of $J_{n}$ and a positive integer $r$ such that

$$
X_{1} X_{k} s^{r}=\sum_{j=1}^{n-1} h_{k j} X_{1} f_{j}
$$

for $1 \leqq k \leqq n$. Thus

$$
X_{k} s^{r}=\sum_{j=1}^{n-1} h_{k j} f_{j}
$$

and

$$
u_{k}[s(u)]^{r}=\sum_{j=1}^{n-1} h_{k j}(u) f_{j}(u)=0 .
$$

Since $s(u) \neq 0$, it then follows that $u_{k}=0$ for each $k$, a contradiction. Hence $A_{n}$ has no basis of $n-1$ elements.

\section{REFERENCES}

1. Hyman Bass, Projective modules over algebras, Ann. of Math. (2) 73 (1961), 532-542.

2. Robert Gilmer and William Heinzer, On the number of generators of an invertible ideal, J. Algebra (to appear).

3. P. J. Hilton and S. Wylie, Homology theory, Cambridge Univ. Press, New York, 1960.

4. R. G. Swan, Vector bundles and projective modules, Trans. Amer. Math. Soc. 105 (1962), 264-277.

Florida State University 